About July 21st Dr. H. C. Semon scraped both hands, and on September 13th injections were again given, beginning with 0.005 c.cm. T.R., reaching 0.12 c.cm. by December 31st, without track reactions or temperature above $98.4^{\circ}$, and the left hand was much improved, there being no open wound and no pain. After 0.23 c.cm. T.R. on January 31st, 1935, the patient had 0.1 c.cm. T.A.F. on February 4 th, followed by no track reaction, but a temperature of $100.2^{\circ}$ and also slight general reaction. The dose on April 15th was $0.35 \mathrm{c.cm}$. T.A.F. The weight on February 22nd, 1934, was 12 st. ; on December $17 \mathrm{th}, 12 \mathrm{st} .11 \mathrm{lb}$. The skin condition before and after treatment is illustrated on the Special Plate.

This patient reported a similar condition on the left arm of his son, aged 32 , appearing just before his own eruption. It extended three inches across the outer side of the arm and three inches above the elbow-joint. It was not red and not raised. The veterinary surgeon treated it, but without change. The son was accidentally shot in September; 1932.

\section{General Conclusion}

The answer to the question, "Will tuberculin cure lupus?" is unknown, but the arrest of disease and the improvement seen in these cases are strong indications that the work should be continued. The fact should be mentioned that in one of the earlier cases (not recorded) seven months' treatment was given before any change in the lesions could be recognized. I have heard a doctor condemn tuberculin after using it on one case for three months only. All the cases treated were of long duration before they received tuberculin. It would be of inestimable value if cases could be treated in the early stage ; we are more likely to learn from early cases than from advanced cases.

F. M. (Case I), notwithstanding the fact that allergy was so marked, continued to improve with small doses. The pertinent question asked me by an expert, "If you get improvement with small doses, why use large ones?" naturally comes into one's mind. I only proceed to large doses if the patient tolerates ther without rise of temperature or unpleasant general reaction. This tolerance varies. One patient will sometimes feel ill after $0.1 \mathrm{c} . \mathrm{cm}$., whereas another will feel no discomfort after $1.6 \mathrm{c.cm}$. I do not look upon any particular dose as the maximum for any tuberculin. The most sensitive case $I$ have yet had has only recently come under treatment ; the patient, a female aged 22, has extensive glandular enlargement in the neck and tuberculides on the hands. As little as $0.0000005 \mathrm{c} . \mathrm{cm}$. of tuberculin sent her temperature up to $101^{\circ} \mathrm{F}$.

Increase of weight almost invariably accompanies administration of tuberculin. Sooner or later one finds the improvement is not as rapid as at first. I now look upon this as a sign for temporary suspension of treatment, or for extension of the interval: in any case it behoves caution. It does not occur when any particular dose has been reached.

Illustrative of the clinical lessons incidental to tuberculin treatment is a case reported in the issue of the Proceedings of the Royal Society of Medicine above mentioned, in which failure to respond to tuberculin in the usual way led to the correction of a wrong diagnosis of "tuberculosis " to the correct one of "syphilis."

Finally, I would call attention to the remarkable differences in the blood films one finds in the different types of cutaneous cases. and suggest that workers should do monthly differential blood counts on each case.

I am indebted to Miss Clark of the $x$-ray department, Royal Northern Hospital, for having the photographs taken, and to Dr. Robert Carswell for advice and assistance in the preparation of this article for publication. Attached is a list of authors whose work I have found useful.

\section{BIBLIOGRAPHY}

Bandelier and Roepke: Tuberculin in Diagnosis and Treatment. Translation by Wilfred B. Christopherson, 1913.

Bredeck, J. B.: " Tuberculin and the Schilling Blood Differential Count in the Diagnosis and Classification of Tuberculosis," American Review of Tuberculosis, April, 1933.

Carswell Robert: Papers on tuberculin, 1933.

Gillespie, John R.: A Rational Method of Using Tuberculin, 1918. Jadassohn, J.: "Remarks on Tuberculin in Dermatology," British Journal of Dermatology and Syphilis, 1929.

Riviere and Morland: Tuberculin Treatment, 1912.

Semon and Burnell-Jones: "Tuberculin in the Treatment of Cutaneous Tuberculosis," British Journal of Dermatology and Syphilis, 1934.

Wilkinson, W. Camac: The Principles of Immunity in Tuberculosis, 1926 ; Tuberculin, Vindicated by Technique, 1933 (the table on page 61 ).

\section{INTUSSUSCEPTION IN THE ADULT DUE TO TUMOURS}

\section{A NOTE ON FIVE CASES}

BY

E. N. MACDERMOTT, B.Sc., M.D., F.R.C.S.I.

HONORARY SURGEON, CENTRAL HOSPITAL ; PROFESSOR OF THERAPEUTICS, UNIVERSITY COLLEGE, GALWAY

(With Special Plate)

Intussusception in the adult is of sufficient rarity to render reports of isolated cases interesting; the occurrence of five in the author's pract:ce in the past year seems, therefore, worthy of report.

\section{Case Reports}

CASE I

A male, aged 28 , was first seen by me on October 10th. 1933, complaining of abdominal pain, vomiting, and constipation for ten days; he stated that he had had similar attacks during the previous three and a half years, and that he had passed blood and "slimy stuff" at intervals. During the two days before I saw him he had received three enemata, with one constipated and two "coloured" results. On examination he had a temperature of $97.8^{\circ}$ and a pulse of 100 . His abdomen was distended and resonant to percussion; the distension made accurate palpation impossible, but there was a sensation of resistance in the right side. A tentative diagnosis of intestinal obstruction was made, and the abdomen was opened through a right paramedian incision under ether anaesthesia. On opening the peritoneum some blood-stained fluid escaped. An intussuscepted mass was found in the right hypochondrium, small gut entering large gut, and caecum and appendix not being visible. The small gut was enormously dilated, almost to the size of the colon, outside the intussusception. The latter was reduced, when it was found that an originally enteric intussusception had passed through the ileocaecal valve and, carrying this along with it, had intussuscepted the caecum and appendix into the ascending colon. The whole mass was about eight inches long. There was a polyp about nine inches above the ileocaecal junction; this polyp had occupied the apex of the intussusception. Above the polyp the bowel was difated to about the diameter of three fingers, while below it was collapsed and contracted to the size of the little finger. As resection and anastomosis did not seem advisable, even if feasible, a McBurney incision was made on the right side, and the polyp, with about three inches of gut on each side of it, extruded and resected after suturing the two limbs to each other and to the peritoneum ; clamps were left on the intestine until the following day. Before closing the abdomen one other polyp was located in the small gut and demonstrated to the assistant. The abdomen was then closed.

Post-operatively the patient did very well, but there was a tendency for the gut of the proximal segment to prolapse through the enterostomy opening, which interfered with spontaneous closure. In mid-November the redundant bowel was resected and the spur clamped. Faeces were passed per rectum on November 24th. On December 9th an attempt was mado 
to suture the muccsa of the fistula; this failed. The skin in the neighbourhood of the fistula was so unhealthy as to preclude any attempt at closure before January 13th, when, under general anaesthesia, the fistula was excised by an elliptical incision prolonged upwards and downwards, and the abdominal wall dissected into layers; the peritoneum was opened, the opening into the intestine excised, and an endto-end anastomosis done. The appendix, which was bulbous, was removed; it contained a polypus (Fig. 1) and some shot in its distal portion. The abdominal wall was closed with a small rubber dam drain down to the peritoneum ; it healed rapidly, the patient leaving hospital four weeks later.

No attempt was made to remove further polypi as the patient had had quite enough tax on his strength in the closure of the fistula. On February 8th a barium meal follow through was carried out, but the radiologist was unable to obtain any evidence of a polyp down as far as the descending colon; this failure was interesting in view of the definite demonstration of a small gut polyp at the first operation. The patient has remained free from symptoms to date (November, 1934), but his sister, who complained of passing blood and mucus per rectum, has been under the care of a physician; this is again of interest, as a familial incidence of intestinal polyposis has been described.

\section{CASE II}

A woman, aged 74, was referred under a tentative diagnosis of appendix abscess on November 6th, 1933. She had suffered from constipation for several months, and for ten days before admission had complained of abdominal pain and vomiting, at first unaccompanied by fever, but when seen the pulse rate was 112 and the temperature $99.4^{\circ}$. The pain, which had been at first generalized, was now referred to the right iliac fossa. The patient was a very fat multipara, and hence accurate abdominal examination was out of the question. There was a feeling of increased resistance, not actually amounting to rigidity, on the right side of the abdomen and tenderness on pressure in the right iliac fossa. She gave no history of diarrhoea or of bloody stools; rectal examination was negative. She had definite auricular fibrillation.

Under ether anaesthesia the abdomen was opened by a Davis incision. The small gut was intussuscepted into the large gut for about eight inches. The intussusception was reduced, and a carcinoma of the caecum was found at the apex of the intussusceptum. The bowel was in good condition. The caecum, three inches of ascending colon, and about four inches of ileum were resected by Mikulicz's method, a tube being inserted in the ileum ; the "gun-barrel " was stitched to skin as well as to the peritoneum, as there was considerable tendency to retraction. For ten days the patient progressed favourably, and then cardiac failure developed and she died on the twelfth day after operation.

\section{CASE III}

A woman, aged 35, had complained for "over a year" of attacks of pain, vomiting, and constipation. For nine days prior to admission she had general abdominal pain, vomiting, and absolute constipation. On examination the abdomen was distended and a slightly tender mass was palpable in the right lumbar region. Under ether anaesthesia the abdomen was opened by a right paramedian incision; an enterocolic intussusception was delivered and reduced. A tumour, which had occupied the apex of the intussusceptum, was palpable within the caecum on its outer side: the caecum, with about three inches of ileum and four inches of the ascending colon; was resected by Mikulicz's method, the mass being exteriorized through a right McBurney incision. The patient died thirty-six hours later of a pulmonary embolus. The tumour was a lipoma of the caecum.

Note.-The tumour, which is shown in Fig. 2, was greyishyellow in colour, and it was attached to the outer wall of the caecum by a pedicle; on section it had a firm, fibrous capsule beneath the coating it derived from the caecal mucosa, and presented the typical appearance of a lipoma. Lipomata of the gastro-intestinal tract are rare ; 242 cases are reported by Poston (1934), of which eighty occurred in the caecum ; there was intussusception in eighty cases altogether, of which six were caecal. From these figures intussusception is no more likely to complicate caecal lipomata than similar tumours in any part of the intestine.

\section{CASE IV}

A man, aged 41, was first seen on April 14th; 1934, when he gave a history of pain in the hypogastric and left iliac regions accompanied by constipation and the passage of blood and mucus in the stools for four months. On rectal examination blood and mucus were found, also a soft mass, which was only just palpable by the finger-tip; no attachment to the rectal wall could be felt by the finger. The patient was prepared for sigmoidoscopy, but after the enema no mass was palpable in the rectum and he felt relieved. He insisted on returning home on April 17th, but came back a week later with pain, constipation, frequent passage of blood and mucus per rectum, and a tendency to vomit. The mass was again palpable per rectum, but abdominal examination, except for some distension, was negative. A provisional diagnosis of polypus of the colon or intussusception was made.

The abdomen was opened on April 26th by a left paramedian incision under ether anaesthesia. An intussusception of the pelvic colon into the rectum was reduced, and a sinall scirrhus of the pelvic colon was found just behind the apex of the intussusceptum. After mobilization, the growth, with a loop of colon, was exteriorized through a left McBurney incision, and the two limbs of the loop stitched together; the primary incision was closed. Three days later the locp was resected. Four weeks later the fistula was about the size of a goose quill, and faeces were being passed by the rcctum. The patient was sent home, hoping for spontaneous closure, but after six weeks he returned; the fistula was of the same size and no faeces were being passed per rectum. Under general anaesthesia the fistula was excised. The upper end of the lower segment was found completely closed, while there was a minute opening in the upper segment; both ends of the colon were opened, and an extraperitoneal end-to-end anastomosis performed. The wound was closed layer by layer and a dam drain left down to the suture line. The patient left hospital eleven days later in apparently perfect condition. The interesting point in this case is that the growth was about one inch behind the apex of the intussusceptum, not at the apex, which was formed by a spastic ring in the colon, apparently due to irritation from the growth.

\section{CASE V}

A man, aged 50, gave a history of constipation with the passage of blood and mucus per rectum for some months, with attacks of abdominal pain and vomiting. The most severe of the attacks had begun three days before admission, and for two days he had also complained of difficulty and pain on micturition. On examination the abdomen was negative except for slight distension ; a hard, craggy mass was palpable per rectum. The finger could not reach sufficiently high to determine its attachments to the bowel wall, but could be passed around between the mass and the rectal mucosa. In diagnosis intussusception was almost ruled out as improbable in view of the previous four cases, and a carcinoma of the rectum was diagnosed.

The abdomen was opened under general anaesthesia by a left paramedian incision with a view to performing Stage 1 of an abdomino-perineal operation. There was a small amount of blood-stained fluid in the peritoneal cavity. A mass, the size of a child's head, was felt almost filling the pelvis, and identified as a colo-rectal intussusception. This was reduced as far as possible but not completely, when a growth was felt in the iliac colon. The loop, with the growth at its apex, was mobilized and exteriorized through a left McBurney incision, and about nine inches resected as in the preceding case. Despite crushing of the spur the fistula refused to close, and five weeks after the primary operation the fistula was excised and an end-to-end extraperitoneal anastomosis done. The patient left hospital a fortnight later (Figs. 3 and 4 show the specimen removed.)

\section{Discussion}

The report of five such cases would give a wholly erroneous notion of the frequency of this condition, since in the same period I have only operated on nine intussusceptions, one of which, in a child, was associated with a polypus. This collection is due to coincidence analogous to the dictum, which has almost attained the standing 
of a natural " law," that " interesting cases always occur in pairs." The most recent report of such an intussusception is by Poston (1934), from which it appears that the condition is much rarer in Oldham than here. He quotes a total of thirty-eight cases in ten years in hospitals serving 250,000, while about seventy cases have been operated upon in Galway in hospitals serving about 180,000 during the same period.

From the standpoint of pathogenesis intussusceptions may be considered in two groups. In the first are those associated with a tumour, often polypoid, situated at the apex of the intussusception, in which the polyp has been dealt with by the bowel below it as if it were the usual intestinal contents, and hurried along the gut, dragging with it the intestine above.

In the second group are those cases in which there is no pathological change demonstrable in the bowel, or in which the tumour is behind the apex of the intussusception. In this group there has been considerable apparent difficulty in visualizing the mechanism of commencement of intussusception. In this connexion there are two facts which it is necessary to recall : (1) transient intussusception is a naturally occurring movement in the intestine, as shown by Alvarez's cinematographic studies; and (2) it is easy to produce intense local spasm, causing a veritable constriction ring in the intestine either by simply pinching it with a forceps (as I have repeatedly done in the cat and rabbit) or by electrical stimulation (Nothnagel). This constriction ring remains for a considerable time, and it is easy to visualize it as passing into the relaxed distal segment of gut and being grasped there and hustled along as if it were intestinal contents, and so forming the apex of an intussusception. Nothnagel actually claims to have seen an intussusception develop in this fashion during a stimulation experiment. Apparently there is no such spastic ring at the apex of the normally occurring intussusceptions, which accounts for their temporary character. Again, it is reasonable to suppose that the irritation due to a tumour in the bowel, a particle of undigested food, etc., was as adequate an irritant as the momentary pinch by a forceps in causing such a spastic ring to form.

So much has been written about the treatment of intussusception that only brief comment is desirable. It is now almost generally accepted that in face of a definite diagnosis palliative treatment has no place. In the adult, however, the diagnosis is rarely definite and enemata are often used and, as in Case IV, may cause reduction, though the intussusception may recur. When this condition is found at operation it should be reduced as far as possible, and then the extent of bowel to be resected considered; resection is, of course, not called for if the bowel is viable and no tumour is present.

In cases such as the five described I believe resection should always be done, and usually by Mikulicz's method. This latter technique seems to me preferable, for apart from the fact that the disparity in size between the dilated upper and the collapsed lower segment of gut usually renders end-to-end anastomosis impossible, the intestinal wall is so unhealthy as to be but poor material for suture, and the toxic contents of the obstructed bowel are a sorry mess to allow to pass over a recent suture line, even in healthy gut. Also in such cases some form of enterostomy is nearly always desirable, and the condition of the patient often calls for the quickest possible conclusion to the operation. In very bad cases Mikulicz's method may be shortened by exteriorizing the gut and making an enterostomy in the upper segment, leaving the resection of the loop to be completed later ; in practice resection after exteriorization requires no extra anaesthesia and adds so little time that it is hard to imagine a case so bad as to need this division.. Unfortunately this technique is not applicable to children, in whom a faecal fistula is said to be invariably fatal, while an occasional patient recovers after resection; in a child with a non-gangrenous intussusception due to a polyp it might be suggested that after reduction an enteropexy should be done to prevent recurrence, and the removal of the tumour be left to a later date.

No attempt is made to quote extensive literature: Poston gives a bibliography, mainly, however, of intestinal lipoma.

\section{Summary}

1. Five cases of intussusception in the adult due to tumour are described.

2. An attempt is made to explain the mode of origin of intussusception.

3. Reasons are given for preferring Mikulicz's resection in such cases.

Note.-It is a matter for regret that specimens from Cases I, II, and IV were mislaid in the Pathology Laboratory.

\section{Bibliography}

Iason and Filberbaum: Ann. of Surg., 1931, xciii, 1191

Moore: New England Journ. Med., 1932, cvii, 395.

Poston: Brit, Journ. Surg., 1934, xxii, 108.

Poston: Brit. Journ. Surg., 1934, Xxi1,

Cuff and Gosden: Brit. Journ. Surg., 1935, xxii, 623. (Reported sirce paper written.)

\section{ULCERATIONS IN THE STOMACH AFTER ADRENALECTOMY}

BY

\section{J. FREUD, D. LUWISCH, AND F. OESTREICHER \\ (With Special Plate)}

Gibelli, $^{1}$ Elliott, ${ }^{2}$ Stuart and Rogoff, ${ }^{3}$ and others have described the occurrence of gastric ulceration after adrenalectomy in the dog and the cat. Similar ulceration has been noted following extirpation of the pituitary or lesion of the hypothalamus (by Cushing, and by Keller, Hare, and $\mathrm{D}^{\prime}$ Amour ${ }^{4}$ ). The problem so raised is, in our opinion, sufficiently important to justify the presentation of our own confirmatory findings.

\section{Experiment}

In the last five members of a larger series of dogs in which two-stage bilateral adrenalectomy had been performed, we directed our attention to the state of the alimentary mucous surfaces. The animals were sectioned immediately or almost immediately after death, which occurred, after heavy prostration, within twenty-four to thirty hours of the second operation. The interval between the removal of the first and second adrenal varied from twenty to thirty-five days. One of the five animals was kept alive on cortin for two months. Cortin was then withdrawn, despite which the dog survived, and in the course of the next three months developed a colossal adiposity. Incomplete adrenalectomy was suspected, and an " autopsy" performed in vivo under very cautious ether narcosis, despite which the animal succumbed in a manner characteristic of adrenal insufficiency. Exploration of the abdominal cavity failed to reveal any residual adrenal tissue. In the gastric mucosa three or four slight surface depressions were visible macroscopically without any actual ulceration, but they were obviously thinned in comparison with the surrounding tissue. The picture rather suggested healing of previous ulcers.

The remaining four animals all showed gastric mucosal defects of varying size and number. The largest varied in diameter from 3 to $7 \mathrm{~mm}$., the depth usually corresponding to the thickness of the mucosa, though sometimes only the superficial layer was dehiscent. The deeper ulcers resembled a classical gastric ulcer (ulcerus rotundum), 
E. N. MACDERMOTT: INTUSSUSCEPTION IN THE ADULT DUE TO TUMOURS

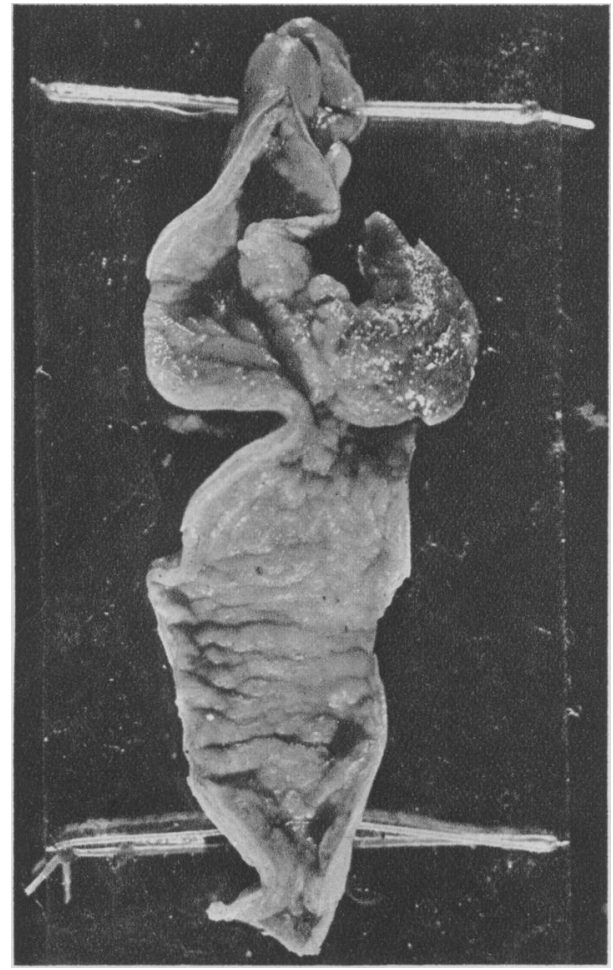

Fia. 1.-Appendix containing polyp removed from Case I

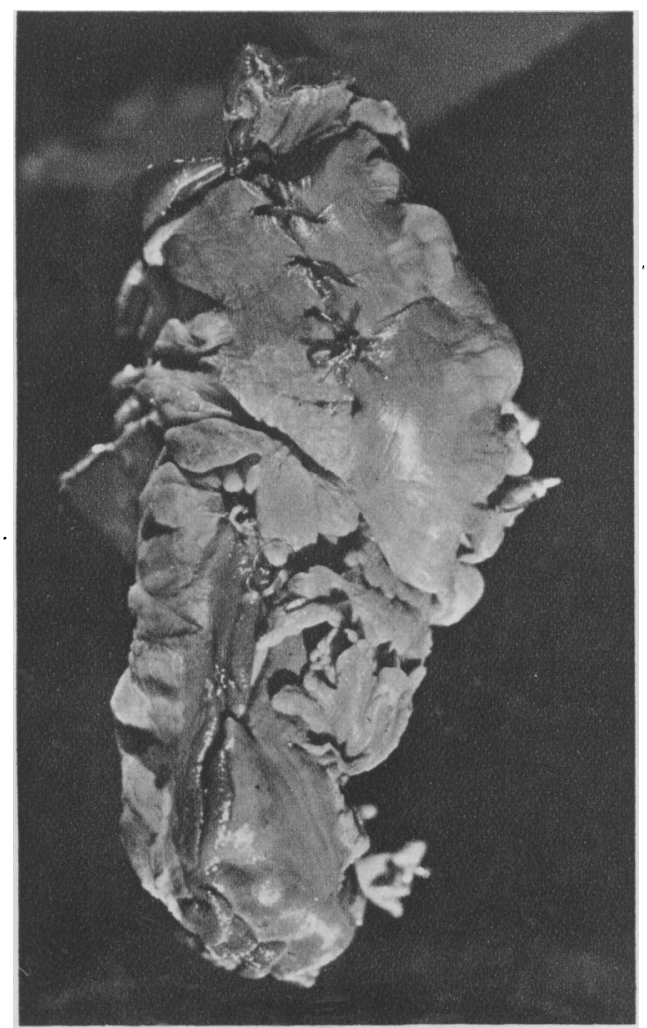

Fra. 3.- Specimen removed from Case $\mathrm{V}$; incompletely reduced intussusception due to cancer of colon.

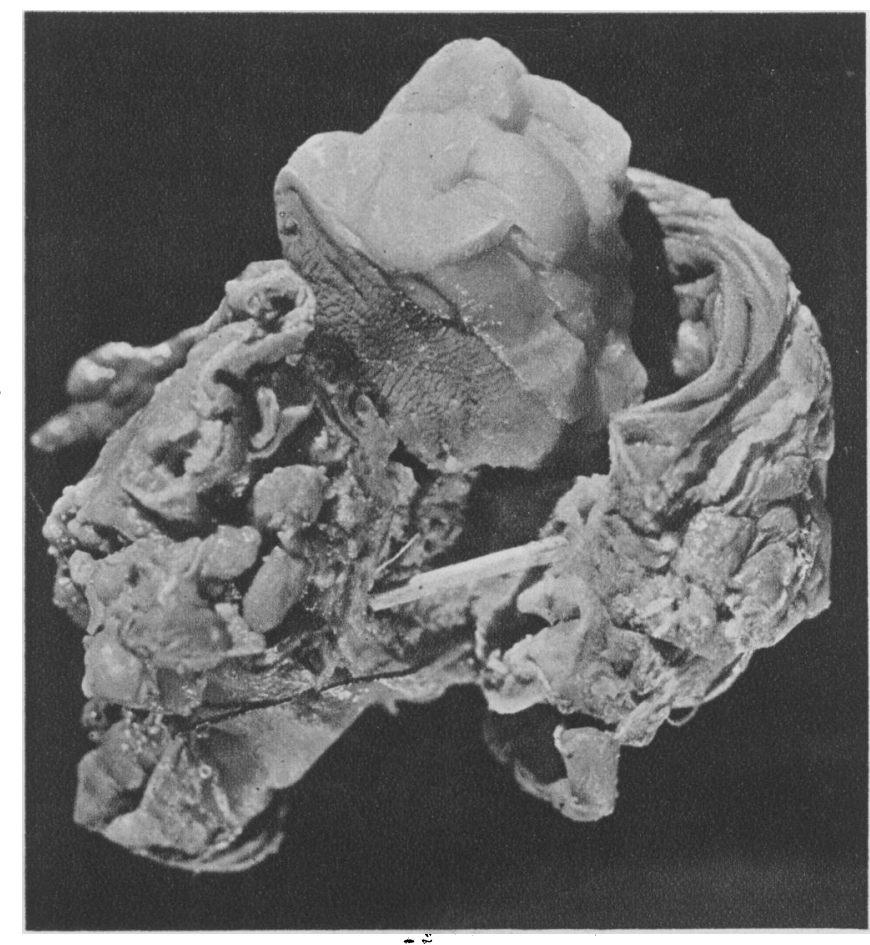

Fra. 2.-Lipoma of caecum removed from Care III

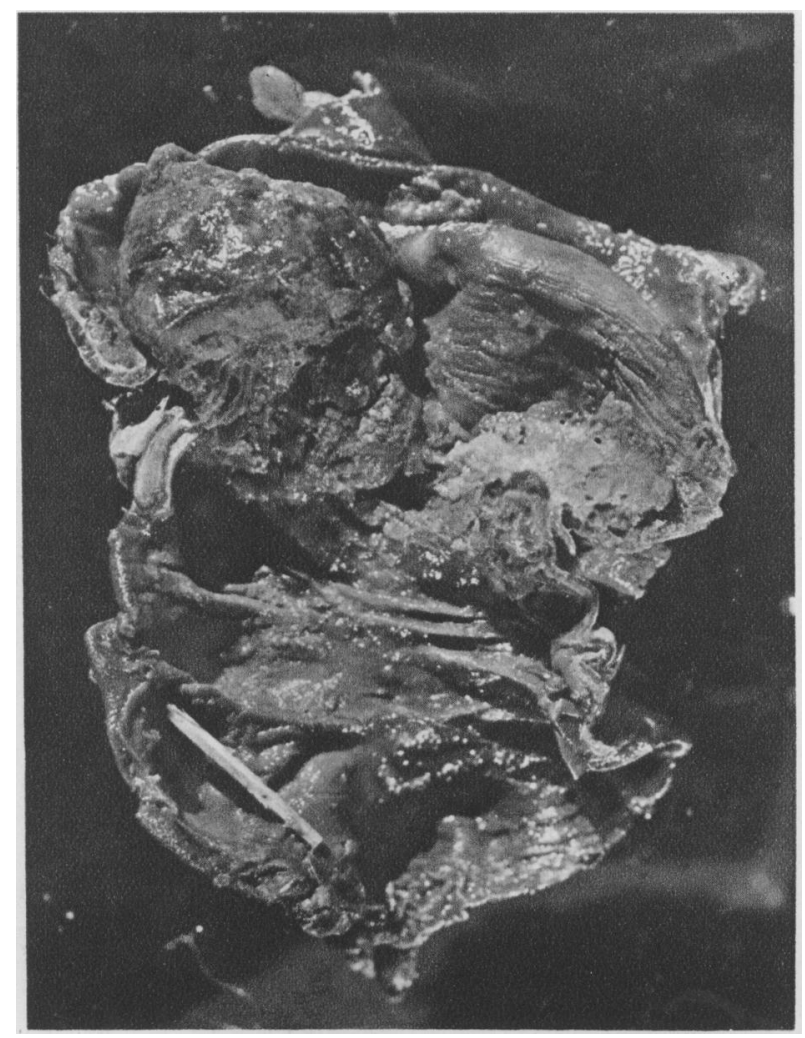

Fig. 4. - Specimen illustrated in Fig. 3 opened to show growth. 